\title{
Laparoscopic pancreaticoduodenectomy in pancreatic ductal adenocarcinoma
}

\author{
Munseok Choi ${ }^{3}$, Seoung Yoon Rho ${ }^{3}$, Sung Hyun Kim, ${ }^{1,2}$, Ho Kyoung Hwang ${ }^{1,2}$, Woo Jung Lee ${ }^{1,2}$, Chang Moo Kang ${ }^{1,2}$ \\ 'Division of Hepatobiliary and Pancreatic Surgery, Department of Surgery, Yonsei University College of Medicine, Seoul, Korea \\ 2Pancreatobiliary Cancer Center, Yonsei Cancer Center, Severance Hospital, Seoul, Korea \\ ${ }^{3}$ Department of Surgery, Yongin Severance Hospital, Yonsei University College of Medicine, Yongin, Korea
}

\begin{abstract}
Laparoscopic pancreatoduodenectomy (LPD) in pancreatic cancer is primarily criticized for its technical and oncological safety. Although solid evidence has not yet been established, many institutions are performing LPD for pancreatic cancer patients, with continuous efforts to ensure oncologic safety. In this video, we demonstrated a case of standard LPD combined with vascular resection in pancreatic cancer.
\end{abstract}

Keywords: Laparoscopy, Pancreatic neoplasms, Pancreatoduodenectomy, Vascular surgical procedures
Received June 17, 2021 Revised August 24, 2021

Accepted August 26, 2021

Corresponding author Chang Moo Kang

Department of Surgery, Yonsei University College of Medicine, \#201 Ludlow Faculty Research Building, 50 Yonsei-ro, Seodaemun-gu, Seoul 03722, Korea

Tel: $+82-2-2228-2135$

Fax: +82-2-313-8289

E-mail: cmkang@yuhs.ac ORCID:

https://orcid.org/0000-0002-5382-4658

This video was presented at the 2020 Asian Congress of Robotic and Laparoscopic Surgery.

Copyright $\odot$ The Korean Society of Endoscopic and Laparoscopic Surgeons.
This is an Open Access article distributed under the terms of the Creative Commons Attribution Non-Commercial License (http:// creativecommons.org/licenses/by-nc/4.0/) which permits unrestricted non-commercial use, distribution, and reproduction in any medium, provided the original work is properly cited.

\section{INTRODUCTION}

Laparoscopic pancreatoduodenectomy (LPD) remains a controversial treatment method for pancreatic ductal adenocarcinoma, as laparoscopic resection followed by reconstruction of the resected bile duct and pancreatic remnants should be performed. In pancreatic head cancers, laparoscopic resection of the pancreaticoduodenal unit could be quite difficult due to potential tumor invasion near the major vascular structures. Moreover, it involves a risk of postoperative complications, such as pancre- atitis and cholangitis. Therefore, it is difficult to ensure patient safety and the completion of the procedure [1].

However, the recent National Comprehensive Cancer Network guidelines recommend minimally invasive surgical treatment, such as laparotomy, for resectable pancreatic cancer. However, only level 3 evidence is currently available regarding LPD for pancreatic head cancers. No prospective randomized control trial (RCT) has compared LPD and open pancreatoduodenectomy (PD) in terms of the short-term and long-term oncologic outcomes for pancreatic cancer because of the following reasons: (1) 
LPD for pancreatic cancer is technically difficult, (2) only a few qualified surgeons are available, and (3) a very small proportion of patients with pancreatic cancer are eligible for this intervention.

In this article, a surgical approach to LPD for pancreatic head cancer was described, and recent updates on short-term oncologic outcomes were briefly summarized.

\section{MATERIALS AND METHODS}

We previously described our surgical LPD technique for periampullary tumors [2,3]. In this section, additional oncologic concepts are introduced to develop a surgical strategy for LPD. Finally, our institutional experiences with LPD for pancreatic head cancer are presented. The relevant video clip is provided with this article (Supplementary Video 1).

\section{Extent of surgery}

Several important prospective RCTs have determined the optimal extent of surgical resection for pancreatic cancer [4]. Standard PD was not considered inferior and had comparable oncologic outcomes to LPD in treating resectable pancreatic cancers. Hence, LPD with standard dissection is considered technically available, feasible, and safe for well-selected pancreatic head cancers.

\section{Superior mesenteric artery lateral margin}

The pancreatic neck margins, retroperitoneal margins, and superior mesenteric artery (SMA) lateral margin should be carefully obtained during PD, as pancreatic cancer cells, regardless of location, can invade and infiltrate pancreatic nerve tissues leading to the major arteries-the SMA, common hepatic artery, and celiac axis [5]. Recently, we reported the potential application of indo- cyanine green (ICG) to secure the SMA lateral margin during LPD. ICG $\left(5 \mathrm{mg} / 2 \mathrm{~cm}^{3}\right)$ was prepared and intravenously injected when dissecting uncinate process of the pancreas [6].

\section{Combined vascular resection}

PD combined with venous resection (VR) is no longer contraindicated for pancreatic head cancer. However, patients who underwent PD-VR exhibited higher rates of complications, including in-hospital and 30-day mortality [7]. Therefore, LPD-VR needs to be performed by experienced surgeons in eligible patients. Various techniques of VR (tangential vs. segmental) and reconstruction (end-to-end vs. inter-position graft) have been reported, but not concluded which one is superior on perioperative outcome [8]. Despite demonstrating the safety and feasibility of LPD with combined resection of the superior mesenteric-splenic-portal venous confluence in treating pancreatic cancer, we acknowledge the difficulty in performing this technique. Therefore, we prefer venous tangential resection to segmental resection in LPD for pancreatic cancer to enhance patient safety (Fig. 1).

\section{RESULTS}

\section{General characteristics of patients}

From June 2014 to November 2020, the medical records of 50 patients, of which 44 underwent LPD and six underwent robotassisted LPD for pancreatic head cancer were collected and reviewed. The clinical characteristics of the patients are summarized in Table 1.

\section{Perioperative outcomes}

The perioperative outcomes are summarized in Table 2. The mean operation time was $483.7 \pm 68.7$ minutes and the mean
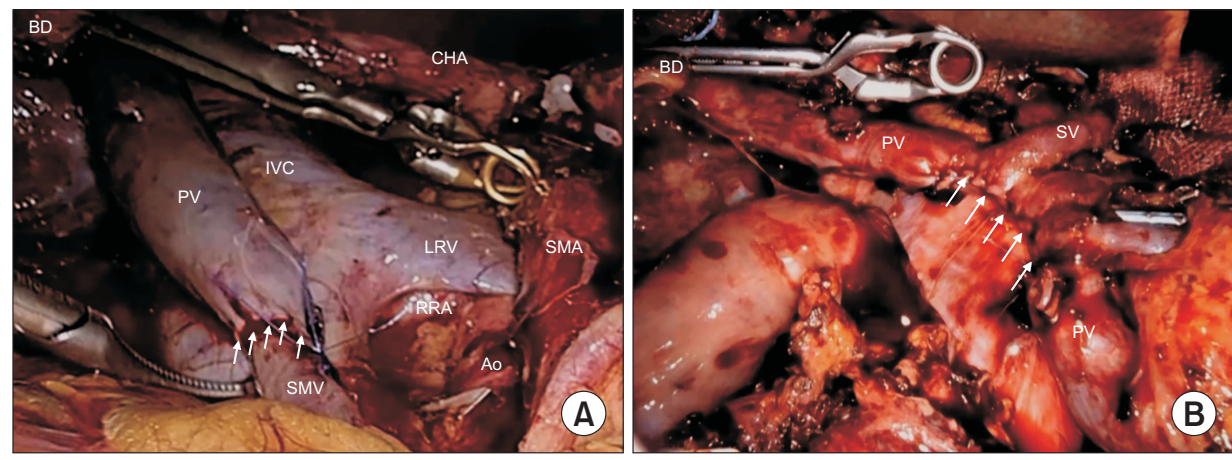

Fig. 1. Laparoscopic view after resection of pancreatic head cancer. (A) Segmental resection of the portal vein (PV) and end-to-end anastomosis, anastomosis site (white arrows). (B) Tangential resection of the PV, stump of resection (white arrows). BD, bile duct; SMA, superior mesenteric artery; CHA, common hepatic artery; LRV, left renal vein; SMV, superior mesenteric vein; Ao, aorta; IVC, inferior vena cava; SV, splenic vein. 
estimated blood loss was $419.4 \pm 461.5 \mathrm{~mL}$. The mean pancreatic duct size was $4.6 \pm 3.1 \mathrm{~mm}$. Among the patients, nine (18.0\%) underwent LPD-VR, 20 (40.0\%) had a soft pancreas, and two patients (4.0\%) developed a postoperative pancreatic fistula.

\section{Short-term oncologic outcomes}

Table 3 shows the pathological characteristics. In the overall cohort, the mean tumor and lymph node sizes were $2.5 \pm 1.0 \mathrm{~cm}$ and $15.1 \pm 9.5 \mathrm{~cm}$, respectively. Among the patients, 44 (88.0\%) had R0 status, five (10.0\%) underwent R1 resection, and one (2.0\%) underwent $\mathrm{R} 2$ resection.

\section{DISCUSSION}

LPD for pancreatic head cancer has theoretical advantages. First, a minimally invasive surgery can enhance postoperative recovery, thereby preserving the patient's functional capacity and initiating adjuvant chemotherapy promptly. Second, LPD has frequently reduced intraoperative bleeding, thus lowering the risk of an intraoperative transfusion, which is known to adversely impact patient survival [9].

Although margin-negative radical pancreatectomy is most effective in treating pancreatic cancer, more than $80 \%$ of pancreatic cancer patients have unresectable tumors at the time of diagnosis. However, considering future advancements facilitating early diagnosis, the number of stage I and stage II patients, having tumors confined to the pancreas, is expected to increase. Therefore, the oncologic role of LPD should be reevaluated be-

Table 1. General characteristics of patients

\begin{tabular}{|cc|}
\hline \multicolumn{1}{|c}{ Characteristic } & MIPD \\
\hline No. of patients & 50 \\
\hline Sex, male/female & $21(42.0) / 29(58.0)$ \\
\hline Age $(\mathrm{yr})$ & $66.3 \pm 8.8$ \\
Preoperative BMI $\left(\mathrm{kg} / \mathrm{m}^{2}\right)$ & $23.4 \pm 2.5$ \\
ASA PS classification & \\
I & $2(4.0)$ \\
II & $17(34.0)$ \\
III & $31(62.0)$ \\
Preoperative CA 19-9 (U/mL) & $227.4 \pm 435.4$ \\
Neoadjuvant CCRT & $7(14.0)$ \\
\hline
\end{tabular}

Data are expressed as number only, mean \pm standard deviation, or number (\%).

MIPD, minimally invasive pancreatoduodenectomy; BMl, body mass index; ASA, American Society of Anesthesiologists; PS, physical status; CA 19-9, carbohydrate antigen 19-9; CCRT, concurrent chemoradiotherapy. cause most patients with stage I and II pancreatic cancer could undergo LPD using the described surgical strategy.

We have previously reviewed the potential feasibility of LPD for pancreatic head cancer [10]. We found that LPD can provide equivalent or superior short-term oncologic outcomes and comparable long-term survival outcomes to those of the standard procedure, suggesting its technical feasibility and oncologic safety as a treatment method [3]. Although RCTs are regarded as the highest level of evidence, other circumstances should be considered. We therefore acknowledge some skepticism on whether RCTs can serve as the fundamental basis in choosing the appropriate treatment for patients. This is true for complex treatment modalities, such as LPD. Therefore, despite the retrospective study design, previous experiences and decisions of expert surgeons on treating their patients should still be considered. However, a successful RCT could be useful in choosing an appropriate surgical approach for pancreatic cancer patients.

Table 2. Perioperative outcomes

\begin{tabular}{|c|c|}
\hline Characteristic & $\operatorname{MIPD}(n=50)$ \\
\hline Operation time (min) & $483.7 \pm 68.7$ \\
\hline Estimated blood loss (mL) & $419.4 \pm 461.5$ \\
\hline Intraoperative transfusion & $2(4.0)$ \\
\hline \multicolumn{2}{|l|}{ Pancreas texture } \\
\hline Soft & $20(40.0)$ \\
\hline Hard & $28(56.0)$ \\
\hline Pancreas duct size (mm) & $4.6 \pm 3.1$ \\
\hline \multicolumn{2}{|l|}{ POPF grade } \\
\hline None & $34(68.0)$ \\
\hline Biochemical leak & $14(28.0)$ \\
\hline B & $1(2.0)$ \\
\hline C & $1(2.0)$ \\
\hline \multicolumn{2}{|l|}{ DGE grade } \\
\hline None & $45(90.0)$ \\
\hline A & $4(8.0)$ \\
\hline B & $0(0)$ \\
\hline C & $1(2.0)$ \\
\hline Postoperative hemorrhage & $0(0)$ \\
\hline Vascular resection & $9(18.0)$ \\
\hline Open conversion & $4(8.0)$ \\
\hline Adjuvant chemotherapy & $34(68.0)$ \\
\hline Hospital stay (day) & $22.5 \pm 18.6$ \\
\hline
\end{tabular}

Data are expressed as mean \pm standard deviation or number (\%). MIPD, minimally invasive pancreatoduodenectomy; POPF, postoperative pancreatic fistula; DGE, delayed gastric emptying. 
Table 3. Short-term oncologic outcome

\begin{tabular}{|cc|}
\hline Variable & MIPD \\
\hline Tumor size $(\mathrm{cm})$ & $2.5 \pm 1.0$ \\
\hline T stage & 44 \\
T1 & $13(29.5)$ \\
T2 & $27(61.4)$ \\
T3 & $4(9.1)$ \\
N stage & 45 \\
N0 & $24(53.3)$ \\
N1 & $14(31.1)$ \\
N2 & $7(15.6)$ \\
Retrieved LN (cm) & $15.1 \pm 9.5$ \\
Positive LN (cm) & $1.4 \pm 2.3$ \\
Invasion & 49 \\
Lymphovascular & $20(40.8)$ \\
Perineural & $42(85.7)$ \\
R status & 50 \\
0 & $44(88.0)$ \\
1 & $5(10.0)$ \\
2 & $1(2.0)$ \\
\hline
\end{tabular}

Data are expressed as mean \pm standard deviation, number only, or number $(\%)$.

MIPD, minimally invasive pancreatoduodenectomy; LN, lymph node.

Thus, we recommend a more careful clinical investigation into the potential application of LPD in pancreatic cancers.

\section{NOTES}

\section{Ethical statements}

The study was conducted in compliance with the principles of the Declaration of Helsinki and the study protocol was reviewed and approved by Institutional Review Board (IRB) of Yonsei University College of Medicine (registered on July 16, 2019; registration number: 2019-1411-001). A waiver of informed consent was approved by the IRB.

\section{Authors' contributions}

Conceptualization: $\mathrm{CMK}$

Visualization: WJL, HKH, SHK, SYR

Writing-original draft: $\mathrm{MC}$

Writing-review \& editing: WJL, HKH, SHK, SYR, CMK

All authors read and approved the final manuscript.

\section{Conflict of interest}

All authors have no conflicts of interest to declare.

\section{Funding/support}

None.

\section{ORCID}

Munseok Choi, https://orcid.org/0000-0002-9844-4747

Seoung Yoon Rho, https://orcid.org/0000-0002-1265-826X

Sung Hyun Kim, https://orcid.org/0000-0001-7683-9687

Ho Kyoung Hwang, https://orcid.org/0000-0003-4064-7776

Woo Jung Lee, https://orcid.org/0000-0001-9273-261X

Chang Moo Kang, https://orcid.org/0000-0002-5382-4658

\section{Supplementary materials}

Supplementary materials can be found via https://doi.org/10.7602/ jmis.2021.24.3.169.

\section{REFERENCES}

1. Kang CM, Lee SH, Chung MJ, Hwang HK, Lee WJ. Laparoscopic pancreatic reconstruction technique following laparoscopic pancreaticoduodenectomy. J Hepatobiliary Pancreat Sci 2015;22:202-210.

2. Navarro JG, Kang CM. Pitfalls for laparoscopic pancreaticoduodenectomy: Need for a stepwise approach. Ann Gastroenterol Surg 2019;3:254-268

3. Choi M, Hwang HK, Rho SY, Lee WJ, Kang CM. Comparing laparoscopic and open pancreaticoduodenectomy in patients with pancreatic head cancer: oncologic outcomes and inflammatory scores. J Hepatobiliary Pancreat Sci 2020;27:124-131.

4. Wang W, He Y, Wu L, Ye L, Yao L, Tang Z. Efficacy of extended versus standard lymphadenectomy in pancreatoduodenectomy for pancreatic head adenocarcinoma. An update meta-analysis. Pancreatology 2019;19:1074-1080.

5. Yi SQ, Miwa K, Ohta T, et al. Innervation of the pancreas from the perspective of perineural invasion of pancreatic cancer. Pancreas 2003;27:225-229.

6. Rho SY, Kim JS, Chong JU, et al. Indocyanine green perfusion imaging-guided laparoscopic pancreaticoduodenectomy: potential application in retroperitoneal margin dissection. J Gastrointest Surg 2018;22:1470-1474.

7. Peng L, Zhou Z, Cao Z, Wu W, Xiao W, Cao J. Long-term oncological outcomes in laparoscopic versus open pancreaticoduodenectomy for pancreatic cancer: a systematic review and meta-analysis. J Laparoendosc Adv Surg Tech A 2019;29:759-769.

8. Kendrick ML, Sclabas GM. Major venous resection during total lapa- 
roscopic pancreaticoduodenectomy. HPB (Oxford) 2011;13:454-458.

9. Kim SY, Choi M, Hwang HK, Rho SY, Lee WJ, Kang CM. Intraoperative transfusion is independently associated with a worse prognosis in resected pancreatic cancer-a retrospective cohort analysis. J Clin
Med 2020;9:689.

10. Kang CM, Lee WJ. Is laparoscopic pancreaticoduodenectomy feasible for pancreatic ductal adenocarcinoma? Cancers (Basel) 2020;12:3430. 